\title{
Impactos del fracking y una mirada del panorama colombiano
}

\section{Fracking impacts and a look at the colombian panorama}

\author{
Martha Vergel ${ }^{+}{ }^{(\mathbb{D})}$ y Lili Becerra*(i) \\ ${ }^{+}$Facultad de Ciencias Agrarias y del Ambiente, Universidad \\ Francisco de Paula Santander Ocaña, Ocaña, Colombia \\ *Departamento de Química, Facultad de Ciencias, Universidad \\ Nacional de Colombia, Bogotá, Colombia
}

\begin{abstract}
Resumen. El ser humano en su afán de desarrollo y producción, no se detiene a analizar las implicaciones que conllevan los métodos e insumos usados para llevar a cabo actividades como el fracking, la cual genera externalidades negativas y pasivos ambientales que afectan al medio ambiente natural y a la sociedad. A través de una amplia revisión bibliográfica y un análisis exhaustivo de investigaciones de los últimos cinco años sobre los aditivos químicos utilizados para la fracturación hidráulica, se obtienen interesantes conclusiones sobre el impacto negativo que generan dichos compuestos a la salud humana y al medio ambiente.
\end{abstract}

Palabras Claves. Fracking; fracturamiento hidráulico; contaminación; calidad del agua.

\begin{abstract}
Due to the increase in industrial activities to improve the levels of economic development of society, environmental water quality has been affected. Hydraulic fracturing is a controversial technology since research indicates that the waste and produced water derived from this activity represents a risk to public health and the natural environment. In this article, a literature review focused on evaluating the approximate composition of wastewater was performed. For this, the research published in the last five years was considered. This exhaustive analysis allowed us to identify the negative impact generated by some compounds used during hydraulic fracturing on human health and the environment.
\end{abstract}

Keywords. Fracking; Hydraulic fracturing; contamination; water quality.

Cómo citar. Vergel, M. y Becerra, L. Impactos del fracking y una mirada del panorama colombiano. Jou. Cie. Ing., vol. 12, no. 1, pp. 264-274, 2020. doi:10.46571/JCI.2020.1.23

Recibido: 17/06/2020 Revisado: 21/07/2020 Aceptado: 26/08/2020

\section{Introduction}

Las necesidades del mundo actual van en constante aumento. El crecimiento exponencial de la población, y los hábitos de consumo insostenibles, son algunos de los múltiples factores que están estrechamente ligados a nuestro estilo actual de desarrollo. Esto implica una imparable demanda de bienes y servicios, lo que ha llevado a crear una dependencia del hombre por los

*e-mail: mcvergelv@ufpso.edu.co 
recursos naturales no renovables como el petróleo, los minerales, y el gas natural, esenciales en la producción de electricidad, transporte, manufacturas químicas y plásticas, entre otros. En consecuencia, este consumo acelerado se verá reflejado en suministros futuros limitados, cada vez más difíciles de obtener, y provocarán, según modelos matemáticos de predicción que emplean la curva de distribución logística conocida como la curva de Hubbert, una disminución de la producción a nivel mundial de combustibles fósiles.

Actualmente se emplean una amplia gama de procedimientos para la consecución de recursos naturales no renovables. En este caso, la tecnología juega un papel importante para encontrar y explotar una acumulación de materia y/o energía como recurso, y ha permitido la explotación de yacimientos que antes se encontraban ocultos [1]. En este sentido, la cantidad de agua empleada en cada paso de la extracción y el procesamiento de los combustibles fósiles ocasionará un aumento en la demanda del recurso natural. Esto trae consigo un incremento en la contaminación del agua, debido a la presencia de sedimentos, sales disueltas, trazas de metales, hidrocarburos, radionúclidos, compuestos orgánicos, y otros desechos sólidos o líquidos arrastrados en las corrientes de agua durante la extracción, y que tienen repercusiones en el hombre y el medio ambiente.

Otro factor importante que rige la explotación de los combustibles fósiles es la propiedad del recurso: acceso a él y control del mismo, lo que trae una connotación política y social colateral. El acceso abierto al uso del recurso natural puede conducir a su sobreexplotación y agotamiento, y favorece principalmente a grandes empresarios, contrario panorama para las comunidades que soportan los riesgos ambientales y sociales asociados a la actividad. Si los recursos son del Estado tal como lo dice el artículo 332: "El Estado es propietario del subsuelo y de los recursos naturales no renovables, sin perjuicio de los derechos adquiridos y perfeccionados con arreglo a las leyes preexistentes"; éstos deberían estar controlados para brindar seguridad económica a corto plazo. Sin embargo, generalmente surgen problemas de eficiencia y sostenibilidad debido al mal manejo del recurso, o a que priman los intereses propios sobre los intereses generales de la sociedad [1].

En este documento de revisión se exponen temas de importancia relacionados con el fracturamiento hidráulico o fracking, resaltando algunas consideraciones generales, un análisis de los compuestos químicos reportados en las aguas producidas y su efecto a la salud, así como una breve discusión en torno a los aspectos legales de esta actividad extractivista.

\section{La era del fracking}

La dependencia de recursos naturales no renovables como el petróleo, ha provocado que los yacimientos se agoten paulatinamente, generando una crisis de abastecimiento, que se refleja en la expansión de las fronteras de exploración de hidrocarburos y en la implementación de nuevas técnicas extractivas como el fracking o fracturamiento hidráulico. Esta técnica se basa en inyectar a través de pozos anteriormente excavados una composición de agua con aditivos químicos y arena a elevadas presiones para lograr que las rocas se fracturen, liberando de esta manera los hidrocarburos que contiene, y a través de sistemas de bombeo se almacenan para su posterior distribución [2].

El fracking ocasiona cambios en las características de la corteza terrestre que se localiza alrededor de los pozos, no solo en las propiedades químicas y biológicas, sino también las físicas, debido a los sismos que se localizan en el rango de magnitud 3 a 4 en la escala de Richter [3]. La sismicidad inducida es el nombre que recibe dicha característica y es debido a dos factores: El primero es por la inyección rutinaria de aditivos qumícos y agua generando microsismos; y el segundo es la introducción bajo tierra del agua residual del proceso, ocasionando sismos de magnitud considerable [4].

Uno de los principales aspectos que origina mayor polémica en la fracturación hidráulica está relacionado con el recurso hídrico. Esto debido a los grandes volúmenes de agua que se usan y que comprometen al desabastecimiento en regiones donde la oferta hídrica es bastante 
limitada [5]. Es posible que se requieran de uno a cuatro millones de galones de agua para fracturar hidráulicamente un pozo y que entre $5 \%$ y $80 \%$ de los fluidos inyectados regresan como agua de retorno dependiendo de las características del pozo [6]. Estos grandes volúmenes de fluidos devueltos presentan desafós en el manejo del agua [7].

El uso del agua no es lo único que preocupa, sino también la contaminación del recurso. Los fluidos cargados de aditivos químicos amenazan a las fuentes subterráneas y superficiales, amenazando la salud de la población y la calidad del medio ambiente [3]. Los fluidos de fracturación hidráulica contienen una mezcla de aditivos orgánicos e inorgánicos, cuyas composiciones varían según la región o el uso de la compañía extractivista [8]. Con el fin de evaluar y comprender las opciones de manejo y eliminación, el destino ambiental y el impacto potencial sobre la salud de las aguas empleadas durante la fractura hidráulica, es necesario realizar una caracterización analítica de tales mezclas. Para esto, se deben considerar tanto los aditivos de fracking como los componentes nativos del terreno, los cuales comprenden elementos radiactivos naturales, metales, hidrocarburos y compuestos orgánicos volátiles y no volátiles.

\section{Composición quḿica del agua producida}

Para la construcción de esta revisión, se tuvieron en cuenta 110 artículos publicados durante los últimos 5 años. En estas investigaciones se realiza la caracterización y cuantificación de los compuestos químicos presentes en las aguas recuperadas luego de realizar el proceso de fractura hidráulica, en regiones aptas para la explotación. El análisis tiene en cuenta 3 grupos de compuestos: elementos radiactivos naturales, elementos químicos (minerales y metales) y, compuestos orgánicos.

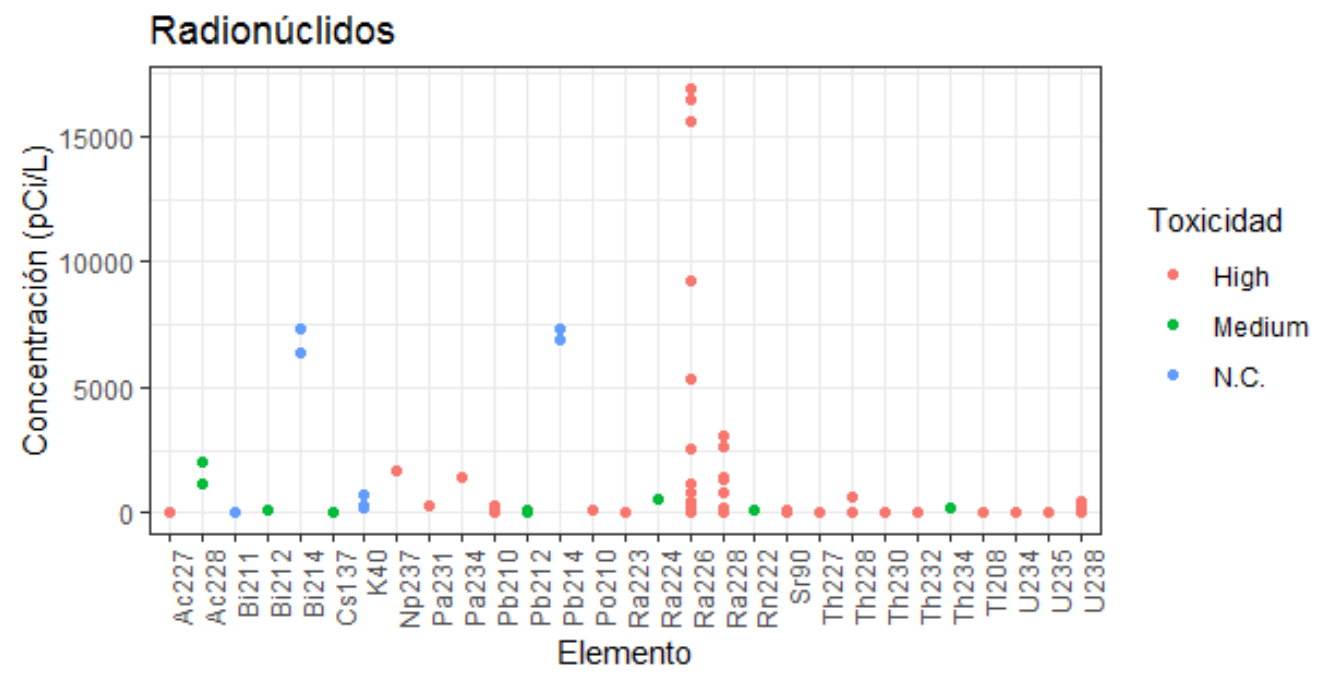

Figura 1: Concentración de materiales radiactivos naturales en aguas producidas. Elaborada a partir de los artículos empleados para la revisión.

En la figura 1, se observa la concentración reportada para los elementos radioactivos encontrados en las aguas producidas de diversos yacimientos. Muchos estudios han reportados niveles significativos de radio, plomo y bismuto. Por ejemplo, los isótopos $\mathrm{Ra}^{226}$ y $\mathrm{Ra}^{228}$ han sido cuantificados en un amplio rango, cuyo valor máximo es de aproximadamente $15000 \mathrm{pCi} / \mathrm{L}$. La Agencia de Protección Ambiental de los Estados Unidos (EPA), recomienda un nivel máximo contaminante (MCL) de $5 \mathrm{pCi} / \mathrm{L}$ en agua destinada al consumo humano, lo cual refleja un alto nivel de exposición a sustancias radioactivas en lugares aledaños a las zonas en las que se adelantan las actividades extractivistas. Aunque es posible que estas concentraciones se 
reduzcan sustancialmente al tratar los efluentes, los valores reportados son muy altos y exceden las regulaciones para compuestos radioactivos [9]. En este sentido, se hace imperante realizar un estudio que evalúe a profundidad, la movilización del material radiactivo natural en las zonas de impacto.

Por otra parte, las concentraciones reportadas para las sustancias inorgánicas y metales, es siempre alta (Figura 2). Estos elementos son parte natural de las aguas producidas, por lo que es normal que elementos como calcio, potasio, y magnesio, se encuentren en altas concentraciones. La salinidad de este tipo de muestras también es alta, por lo que bromo, cloro y sodio son los elementos detectados con mayor frecuencia. El agua producida contiene la misma o mayor cantidad de sales e iones inorgánicos que el agua de mar con una gran cantidad de iones cloruro y sodio [10]. La ingestión oral de estos iones en gran cantidad puede provocar vómitos, irritación del tracto gastrointestinal, dificultad respiratoria, entre otros síntomas [11].

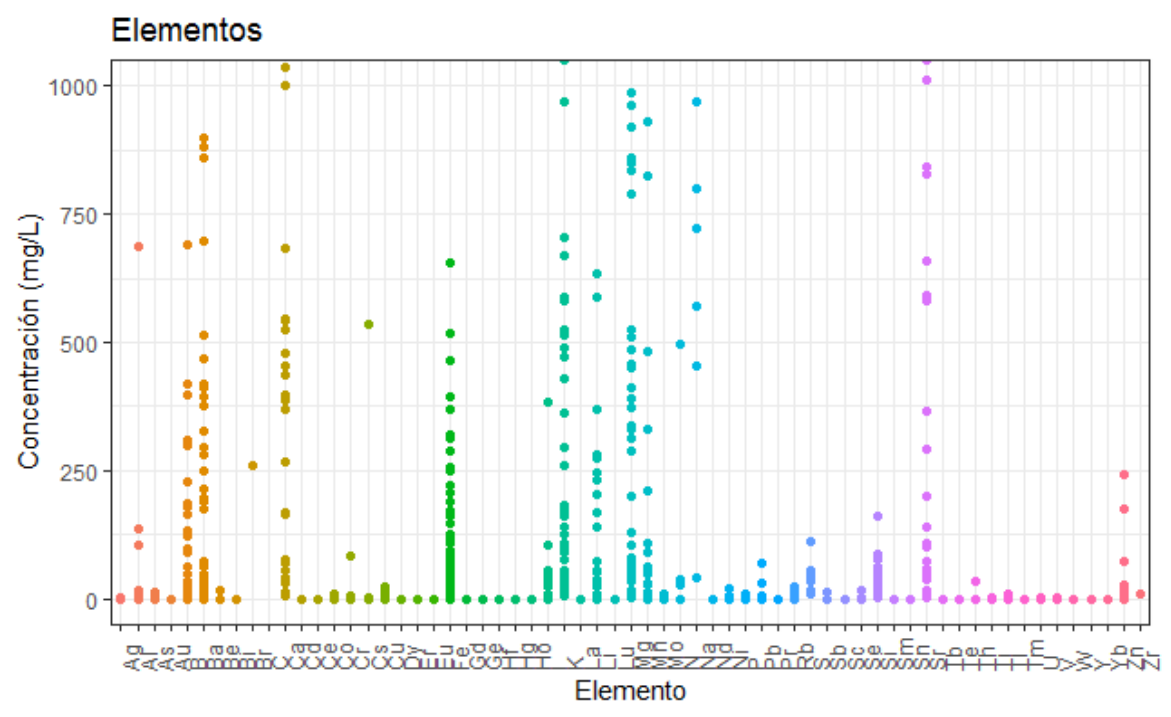

Figura 2: Concentración de minerales en aguas producidas. Elaborada a partir de los artículos empleados para la revisión.

Los metales primarios que se encuentran en el agua producida son bario, hierro, manganeso, cobre y zinc. Si bien la mayoría de los efectos sobre la salud solo se observan con formas solubles de bario, los datos no diferencian las especies químicas. Los efectos de la ingestión de bario incluyen trastornos gastrointestinales y debilidad muscular, vómitos, calambres abdominales, diarrea, dificultades para respirar, aumento o disminución de la presión arterial, entumecimiento alrededor de la cara y debilidad muscular [12]. El plomo es un metal tóxico que ingresa al cuerpo a través de la ingestión, la inhalación y la absorción de la piel y puede acumularse en el tejido. Afecta a la mayoría de los órganos del cuerpo humano, especialmente los riñones y el cerebro [13]. El cromo también es tóxico y el agua está contaminada con cromo, lo que resulta en irritación de la piel, muerte del ganado, etc [14].

Otro aspecto de la calidad del agua producida es su composición orgánica. Se sabe que el carbón contiene varias sustancias orgánicas potencialmente tóxicas, incluídos los hidrocarburos aromáticos policíclicos (PAHs), los compuestos heterocíclicos y las aminas aromáticas. Dado que el agua producida se deriva del agua subterránea que ha estado en contacto con el carbón, esta podría contener sustancias orgánicas tóxicas solubilizadas del carbón [15]. Los PAHs de bajo peso molecular (2 anillos) fueron los compuestos más abundantes encontrados en la mayoría de los lugares estudiados. Por otro lado, los autores reportaron que los hidrocarburos más comunes encontrados en las formaciones de esquisto gaseoso eran más alifáticos que aromáticos [16]. 
Los PAHs son motivo de gran preocupación porque debido a su configuración estructural que tiene quinonas, pueden alquilar fácilmente el ADN, causar defectos de las funciones cardíacas, embriotoxicidad, etc [17]. PAHs específicos como el benzo[a]pireno (BaP), ha sido cuantificado y reportado en investigaciones realizadas por Chittick [18], Bell [19], Flynn [20], Regnery [21], y Shih [22]. La concentración promedio reportada para el BaP, es de aproximadamente $1 \mu \mathrm{g} / \mathrm{L}$. La exposición aguda a altas concentraciones $(>0.2 \mu \mathrm{g} / \mathrm{L})$ puede causar daño a los glóbulos rojos, anemia, función inmunitaria suprimida y efectos sobre el desarrollo y la reproducción [23].

Los compuestos orgánicos caracterizados en las aguas producidas también pueden provenir de los productos químicos orgánicos utilizados para la fracturación hidráulica, por ejemplo, solventes, surfactantes, biocidas, inhibidores de incrustaciones, entre otros [24]. Es importante tener en cuenta que los aditivos químicos utilizados en los fluidos de fracturación hidráulica representan sólo hasta el $0.5 \%$ a $1 \%$ del fluido total utilizado. Sin embargo, estos son los únicos compuestos que pueden usarse como trazadores en el medio ambiente como se discutió anteriormente [8]. En la figura 3, se observan los principales grupos funcionales orgánicos de los compuestos identificados y cuantificados en aguas producidas. Compuestos como el acetaldehído, acetona, ácido fórmico, tioúrea, aminas, amidas o amido-aminas, son empleados como inhibidores de corrosión. Estos compuestos se usan para prevenir la corrosión potencial en la carcasa causada por el uso de ácidos y sales. Otros compuestos como el glutaraldehído y sus derivados, así como una amplia variedad de biocidas, han sido empleados para desinfectar y reducir la concentración de bacterias presentes en los fluidos de fractura, las cuales podrían causar problemas potenciales durante el proceso de extracción, como la obstrucción del pozo, la corrosión del equipo de metal utilizado o incluso la degradación de los productos químicos utilizados en los fluidos [8].

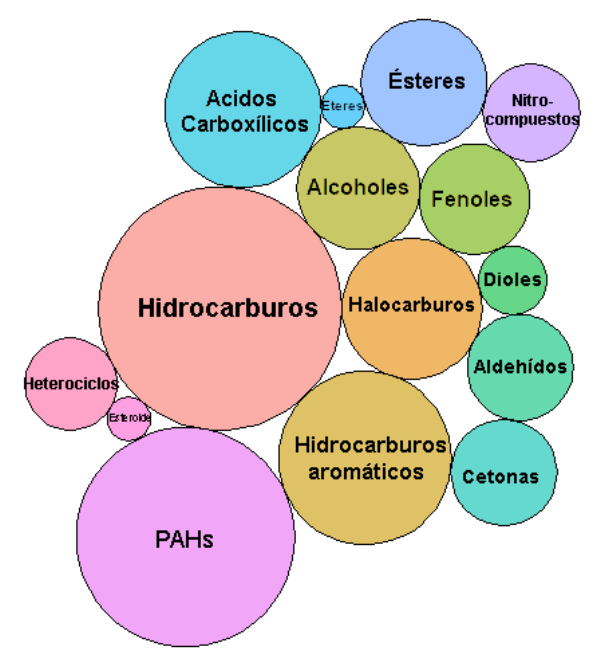

Figura 3: Principales grupos funcionales orgánicos caracterizados en aguas producidas. Elaborada a partir de los artículos empleados para la revisión.

Algunas de las sustancias químicas, como el benceno, el tolueno y el xileno, que se encuentran en el agua producida son carcinógenos conocidos y plantean problemas ambientales y de salud [25]. Se ha informado que el benceno con un nivel máximo de contaminante (MCL) superior a $0.005 \mathrm{mg} / \mathrm{L}$ aumenta los riesgos de cáncer, promueve la anemia y disminuye las plaquetas sanguíneas en las personas afectadas [26]. El consumo de agua contaminada con tolueno (MCL $=1 \mathrm{mg} / \mathrm{L}$ ) aumenta los riesgos de daño renal y hepático [27]. Del mismo modo, se sabe que el consumo de aguas contaminadas con xileno $(\mathrm{MCL}=10 \mathrm{mg} / \mathrm{L})$ causa dolores de cabeza, mareos, falta de coordinación muscular, confusión y cambios en el sentido del equilibrio [28]. Según los 
artículos tenidos en cuenta para esta revisión, 13 de ellos reportan una concentración promedio de $141 \mathrm{mg} / \mathrm{L}$ de benceno, 14 de ellos reportan una concentración promedio de $9 \mathrm{mg} / \mathrm{L}$ de tolueno, y 6 de ellos, reportan una concentraciń promedio de $3 \mathrm{mg} / \mathrm{L}$ de xileno. Esto evidencia una clara necesidad de hacer un buen manejo de las aguas producidas, con el fin de evitar afectaciones a largo plazo.

La fractura hidráulica también conduce a la contaminación del aire con metano, hidrocarburos alifáticos (C2 - C5), alcanos, VOCs, BTEX, sulfuro de hidrógeno, n-hexano y formaldehído [29].Recientemente se ha informado que se pueden liberar 143 contaminantes del aire debido al proceso de fractura hidráulica [30]. Por otra parte, los llamados çontaminantes criterios atmosféricos"son monóxido de carbono, ozono cerca de la superficie, dióxido de nitrógeno, material particulado, dióxido de azufre y plomo. Estos contaminantes, aumentan la incidencia de accidentes cerebrovasculares, enfermedades cardíacas, cáncer de pulmón y enfermedades respiratorias, y es una de las principales causas de muerte y discapacidad en todo el mundo [31]. En Colombia, la resolución No. 2254 del 2017 del Ministerio de Ambiente y Desarrollo Sostenible [32], especifica los niveles máximos permisibles de estos contaminantes.

El interés en la composición del agua producida también está relacionada a un estudio más amplio sobre los efectos a la salud y al medio ambiente de los compuestos empleados durante la extracción de gas y petróleo. La mayoría de los estudios relacionados con la gestión del agua producida se centran en las técnicas de tratamiento del agua producida. Actualmente existen pocos estudios que hagan una evaluación de riesgos para la salud humana y para el ambiente en relación con la descarga de aguas producidas en el medio ambiente natural. Un artículo publicado recientemente por Kabyl [10], integra las medidas de riesgo con el tratamiento del agua producida y las técnicas de reutilización. Para gestionar las descargas de agua producida, los riesgos ambientales, económicos y técnicos deben evaluarse y utilizarse adecuadamente en el proceso de toma de decisiones. Este tipo de esfuerzos son vitales para reducir el riesgo ambiental al enfocar los contaminantes que más contribuyen al riesgo total, e identificando las áreas que deben mejorarse para mitigar el riesgo integrado.

\section{El fracking y la primacía de los intereses políticos}

Las actividades como el fracking generan un escenario complejo en donde confluyen varios intereses, como los políticos, que mantienen conveniencias con el fin de mantener el control de las reservas que aún existen alrededor del mundo [33]. Estos intereses políticos van relacionados con la distribución geográfica de los recursos de hidrocarburos, haciendo que el petróleo siempre tenga un componente político, independientemente de su fungibilidad, y que ha llegado a convertirse en un sistema de interdependencias entre productores, consumidores y países de tránsito [34].

Estas condiciones geográficas, sumadas a las históricas y políticas, se relacionan con los efectos socioeconómicos en un territorio; las diferencias tecnológicas y el grado de dificultad en la extracción del crudo son factores importantes [35]. Al realizar un balance costo-beneficio desde la perspectiva socioeconómica, el resultado es evidentemente negativo: el hidrocarburo proyecta falsas esperanzas en la población cercana al proyecto, que saltan de tiempos de tranquilidad a tiempos de crisis, donde gobiernos o empresas desalojan de sus propios territorios a comunidades indígenas, con la finalidad de explotar los recursos naturales no renovables [35].

La polémica alrededor de la geografía radica en la necesidad de progresar en su integración con la economía y analizar sus interacciones a diferentes niveles en cuanto la afectación al medio ambiente y sociedad. Para Colombia, por ejemplo, no existe una articulación clara entre la política ambiental y las políticas sectoriales. Esto se debe a la falta de una visión integral del territorio, en particular en zonas de explotación petrolera, con gran demanda de empresas multinacionales que buscan altos márgenes de ganancias para mejorar su capital, y tienen en baja consideración la afectación a las comunidades y al medio ambiente [36]. 


\section{Riesgos y controversias}

Además de los impactos ambientales negativos generados por el sector petrolero en sus diferentes etapas (exploración, perforación, explotación, transporte, refinación, comercialización, consumo), existen impactos sociales negativos como las guerras, genocidios, desplazamiento forzado, desestructuración de territorios, crecimiento desordenado de la población, rompimiento del tejido social y la identidad cultural, debilitamiento de las actividades económicas tradicionales, convergencia violenta de grupos armados, legales e ilegales, militarización de la sociedad civil, lucha política por la captura de rentas y la concomitante corrupción en el ejercicio de los cargos públicos [37]. El conflicto armado en Colombia genera una presión y restricción a oportunidades políticas, modificando la evolución del conflicto socio-ambiental [4], pero a su vez provocando caos social y miedo con atentados terroristas en las redes de distribución, característica principal de la guerra en Colombia [38].

No obstante, existen una serie de riesgos que son externos, conocidos como riesgos operacionales. Estos se presentan principalmente como consecuencia de la inflamabilidad del petróleo, lo cual aumenta el riesgo de generar incendios y explosiones, causar daños directos a la infraestructura, provocar derrames sobre el agua, suelo, biota, y ocasionar emisiones contaminantes, lo que da lugar a consecuencias económicas, ambientales y sociales nefastas [39]. Dichas consecuencias ambientales generan afectación directa a la naturaleza y consecuencias graves al medio ambiente, en muchos casos de manera irreversible, afectando el patrimonio natural del país [40].

Representantes del gremio del sector petrolero insisten que sus impactos ambientales negativos son manejables, olvidando los pasivos ambientales que producen luego de terminar las actividades productivas [41]. La rentabilidad del fracturamiento hidráulico no es suficiente para prevenir las numerosas críticas con motivo de las consecuencias medioambientales y sociales que trae consigo la práctica, atentando contra la salud y el desarrollo sostenible en el manejo y aprovechamiento de los recursos naturales [42].

La extracción de petróleo y gas de esquisto ha generado protestas de gran magnitud por los graves problemas ambientales que representa, y es que detrás de estos nuevos mecanismos de extracción se encuentran intereses económicos y políticos, entramados geopolíticos de grandes imperios para la expansión internacional de la explotación [43]. En el caso de Colombia, como los otros países petroleros, admite un dumping ecológico cediendo esos recursos mientras socializa los costos económicos de sus impactos negativos; y es así como en el costo de un barril de petróleo no está contemplado el costo ambiental de los recursos afectados, costo que no le interesa a la empresa ni al Estado [41]. No obstante, al comparar un país como Estados Unidos, con Colombia, el propietario del suelo es también propietario del subsuelo, panorama muy distinto para Colombia, cuyo propietario del subsuelo es el Estado.

\section{Situación actual en colombia}

En Colombia se promueve la inversión extranjera en el sector de hidrocarburos, por medio de mecanismos como el sistema variable de pago de regalías según el tamaño del campo, preferencia de participación hasta de $100 \%$ en proyectos por parte de empresas extranjeras y la creación de las licencias Express (Decreto 2041 de 2014) [44]. El manejo aplicado a la política ambiental de Colombia es contrario a principios constitucionales ambientales, principalmente por el principio de precaución al permitir consideraciones de mitigación a daños ambientales graves [45]. La legislación en Colombia sobre yacimientos no convencionales mediante la técnica del fracturamiento hidráulico, muestra muchos vacíos técnicos y jurídicos, al no contarse con la suficiente información sobre datos hidrogeológicos, ecosistemas sensibles y estudios de estructuras geológicas. En Colombia se ha empezado a introducir posibilidades de ejecución de prácticas de fracturación hidráulica teniendo para esta la misma regulación que se usa para la explotación convencional. No se ha establecido un marco regulatorio específico que abarque las afectaciones 
que este tipo de actividad puede ocasionar al medio ambiente y ecosistemas cercanos a la práctica [46].

La falta de cuerpo normativo para los hidrocarburos no convencionales en Colombia es un problema al no tener representado cada uno de los bienes jurídicos en riesgo, teniendo en cuenta que el fracking es una práctica con mayores impactos y con más complejas técnicas [47]. Evadiendo cualquier concepto técnico, "en febrero de 2019, la Comisión ad hoc de Expertos convocada por el gobierno nacional entregó su informe, en donde conceptuó avalando la posibilidad de explotar yacimientos no convencionales en Colombia y recomendó avanzar con las pruebas piloto de fracking" [48]. Sin importar que Colombia es un país megadiverso, con áreas ambientales únicas y sensibles, características importantes para ser contempladas al momento de realizar una técnica como el fracking [49].

Además, la exploración y explotación de yacimientos no convencionales, requiere la utilizació de tecnologías más costosas, sumado a las tensiones frente a la normatividad y los problemas operativos ambientales [50]. Por lo tanto, se reconoce que energías alternativas como la eólica, solar, mareomotriz, entre otras, son más compatibles con las metas de generación de energía como también con las metas de protección ambiental [4].

La dependencia del ser humano a los combustibles fósiles es una de las principales causas del cambio climático. La elección de otras fuentes de energía es lo ideal [51], siendo necesario escuchar las afirmaciones de los científicos, quienes advierten que para desacelerar el cambio climático es necesario un cambio tecnológico, institucional y humano.Ante la extralimitación en la emisión de gases de efecto invernadero es necesario establecer límites que alerten la necesidad de cambios en el sector petrolero [52].

\section{Conclusiones}

Es necesario repensar actividades como el fracking en países tan susceptibles como Colombia, paś marcado social y ambientalmente. Si bien esta actividad otorga beneficios principalmente a nivel económico, es necesario equilibrar la relación costo-beneficio. Resultados de investigaciones desarrolladas en las zonas de influencia de los sitios dedicados al fracking, reportan la presencia de una amplia gama de compuestos orgánicos con efectos nocivos o tóxicos, tanto para el medio ambiente como para la salud humana y animal, en las aguas producidas y liberadas durante la actividad. Así mismo, el fracking libera de las formaciones geológicas, elementos nativos como metales y elementos radioactivos, los cuales se filtran al agua y al aire provocando efectos adversos, debido a las concentraciones alarmantes en las que han sido hallados. Esto hace evidente la necesidad de realizar evaluaciones de riesgo y monitoreo constante y exhaustivo en las áreas dedicadas al fracking.

Por otra parte, la falta de normatividad específica sobre el fracking en Colombia pone en peligro la sostenibilidad ambiental del territorio, sumado al desconocimiento del mismo, ya que las características biodiversas propias de países como Colombia son incomparables con la de otros territorios donde se ha practicado el fracturamiento hidráulico. Sólo la evidencia científica podrá soportar nuevas reglamentaciones encaminadas a la regulación y gestión de los residuos y los productos químicos y métodos empleados para tal fin. También se deben tener en cuenta otros aspectos importantes, como los culturales y sociales, los cuales son propios de territorios afectados por el conflicto armado, y pueden llegar a ser un obstáculo para el desarrollo de dichas actividades extractivas. Por lo tanto, es necesario tener en cuenta y aplicar principios o regulaciones sólidas para que la producción sea eficaz y se desarrolle minimizando el riesgo para el medio ambiente y la salud humana.

\section{Referencias}

[1] E. Jowsey, "Economic aspects of natural resource exploitation," International Journal of Sustainable Development \& World Ecology, vol. 16, pp. 303-307, 2009/10/12 2009 
[2] A. Maldonado-Torres, B. Estrada-Drouaillet, E. Osorio-Hernández, J.A. López-Santillán, S.G.M. Ravelo, y A. Olvera-Velona, "Fracking... experiencia y perspectivas mundiales," Temas de Ciencia y Tecnología, vol. 21, pp. 59 - 68, 2017.

[3] F. R. Martínez, C. M. Perilla, y J. D. Urquijo, "Fracking: viabilidad económica y ambiental en Colombia" Econografos Escuela de Economía No. 133, Bogotá, Colombia, 2019.

[4] P.B.T. Silva Barbosa y F. Carriazo, "Costos económicos de las externalidades ambientales del fracking: un análisis de metarregresión y algunas implicaciones para Colombia," $R A D$, vol. 21 , n. ${ }^{\circ} 41$, pp. 25-42, may 2018.

[5] S. A. Charry, A. J. Pérez, "Efectos de la estimulación hidráulica (fracking) en el recurso hídrico: Implicaciones en el contexto colombiano," Ciencia E Ingeniería Neogranadina, vol. 28, no. 1, pp. 135-164, 2017.

[6] U.S. EPA (U.S. Environmental Protection Agency), "Hydraulic Fracturing for Oil and Gas: Impacts from the Hydraulic Fracturing Water Cycle on Drinking Water Resources in the United States. Executive Summary.," Office of Research and Development, Washington, DC. EPA/600/R-16/236ES, 2016.

[7] B. D. Lutz, A. N. Lewis, y M. W. Doyle, "Generation, transport, and disposal of wastewater associated with Marcellus Shale gas development," Water Resources Research, vol. 49, pp. 647-656, 2013.

[8] I. Ferrer y E. M. Thurman, "Chemical constituents and analytical approaches for hydraulic fracturing waters," Trends in Environmental Analytical Chemistry, vol. 5, pp. 18-25, 2015/02/01/2015.

[9] IAEA, "A Basic Toxicity Classification of Radionuclides," International Atomic Energy Agency, Technical Report Series 15, Vienna, 1963.

[10] A. Kabyl, M. Yang, R. Abbassi, y S. Li, "A risk-based approach to produced water management in offshore oil and gas operations," Process Safety and Environmental Protection, vol. 139, pp. 341-361, 2020/07/01/ 2020 .

[11] M. C. McLaughlin, T. Borch, B. McDevitt, N. R. Warner, and J. Blotevogel, "Water quality assessment downstream of oil and gas produced water discharges intended for beneficial reuse in arid regions," Science of The Total Environment, vol. 713, p. 136607, 2020/04/15/ 2020.

[12] Long, J., Feinstein, L., Bachmann, C., Birkholzer, J., Camarillo, M., Domen, J., et al., " An Independent Scientific Assessment of Well Stimulation in California Volume II: Potential Environmental Impacts of Hydraulic Fracturing and Acid Stimulations", Lawrence Berkeley National Laboratory. LBNL Report : LBNL-189356, 2015. [En Línea]. Disponible en: https://osti.gov/biblio/1577335. [Accedido: 15-may-2020].

[13] ATSDR (Agency for Toxic Substances and Disease Registry), "Toxicological profile for Lead," Atlanta, GA, U.S. Department of Health and Human Services, Public Health Service, 2020.

[14] ATSDR (Agency for Toxic Substances and Disease Registry), "Toxicological profile for Chromium," Atlanta, GA, U.S. Department of Health and Human Services, Public Health Service, 2012.

[15] W. H. Orem, C. A. Tatu, H. E. Lerch, C. A. Rice, T. T. Bartos, A. L. Bates, et al., "Organic compounds in produced waters from coalbed natural gas wells in the Powder River Basin, Wyoming, USA," SApplied Geochemistry, vol. 22, pp. 2240-2256, 2007/10/01/ 2007.

[16] D. M. Akob, I. M. Cozzarelli, D. S. Dunlap, E. L. Rowan, y M. M. Lorah, "Organic and inorganic composition and microbiology of produced waters from Pennsylvania shale gas wells," Applied Geochemistry, vol. 60, pp. 116-125, 2015/09/01/ 2015.

[17] T. Bakke, J. Klungsøyr, y S. Sanni, "Environmental impacts of produced water and drilling waste discharges from the Norwegian offshore petroleum industry," Marine Environmental Research, vol. 92, pp. 154-169, 2013/12/01/ 2013.

[18] E. A. Chittick y T. Srebotnjak, "An analysis of chemicals and other constituents found in produced water from hydraulically fractured wells in California and the challenges for wastewater management", Journal of Environmental Management, vol. 204, pp. 502-509, 2017/12/15/ 2017.

[19] E. A. Bell, T. E. Poynor, K. B. Newhart, J. Regnery, B. D. Coday, y T. Y. Cath, "Produced water treatment using forward osmosis membranes: Evaluation of extended-time performance and fouling", Journal of Membrane Science, vol. 525, pp. 77-88, 2017/03/01/ 2017.

[20] Y. He, S. L. Flynn, E. J. Folkerts, Y. Zhang, D. Ruan, D. S. Alessi, et al., "Chemical and toxicological characterizations of hydraulic fracturing flowback and produced water", Water Research, vol. 114, pp. 78-87, 2017/05/01/ 2017.

[21] J. Regnery, B. D. Coday, S. M. Riley, y T. Y. Cath, "Solid-phase extraction followed by gas chromatography-mass spectrometry for the quantitative analysis of semi-volatile hydrocarbons in hydraulic fracturing wastewaters", Analytical Methods, vol. 8, pp. 2058-2068, 2016.

[22] J.-S. Shih, J. E. Saiers, S. C. Anisfeld, Z. Chu, L. A. Muehlenbachs, y S. M. Olmstead, "Characterization and Analysis of Liquid Waste from Marcellus Shale Gas Development", Environmental Science Technology, vol. 49, pp. 9557-9565, 2015/08/18 2015.

[23] D. N. Das y S. K. Bhutia, "Inevitable dietary exposure of Benzo[a]pyrene: carcinogenic risk assessment an emerging issues and concerns," Current Opinion in Food Science, vol. 24, pp. 16-25, 2018/12/01/ 2018. 
[24] J. C. McIntosh, M. J. Hendry, C. Ballentine, R. S. Haszeldine, B. Mayer, G. Etiope, et al., "A Critical Review of State-of-the-Art and Emerging Approaches to Identify Fracking-Derived Gases and Associated Contaminants in Aquifers," Environmental Science Technology, vol. 53, pp. 1063-1077, 2019/02/05 2019.

[25] G. J. Getzinger, M. P. O'Connor, K. Hoelzer, B. D. Drollette, O. Karatum, M. A. Deshusses, et al., "Natural Gas Residual Fluids: Sources, Endpoints, and Organic Chemical Composition after Centralized Waste Treatment in Pennsylvania," Environmental Science Technology, vol. 49, pp. 8347-8355, 2015/07/21 2015.

[26] ATSDR (Agency for Toxic Substances and Disease Registry), "Toxicological profile for Benzene," Atlanta, GA, U.S. Department of Health and Human Services, Public Health Service, 2007.

[27] ATSDR (Agency for Toxic Substances and Disease Registry), "Toxicological profile for Toluene," Atlanta, GA, U.S. Department of Health and Human Services, Public Health Service, 2017.

[28] ATSDR (Agency for Toxic Substances and Disease Registry), "Toxicological profile for Xylene," Atlanta, GA, U.S. Department of Health and Human Services, Public Health Service, 2007

[29] D. T. Allen, "Emissions from oil and gas operations in the United States and their air quality implications," Journal of the Air 85 Waste Management Association, vol. 66, pp. 549-575, 2016/06/02 2016.

[30] E. G. Elliott, A. S. Ettinger, B. P. Leaderer, M. B. Bracken, and N. C. Deziel, "A systematic evaluation of chemicals in hydraulic-fracturing fluids and wastewater for reproductive and developmental toxicity," Journal of Exposure Science \& Environmental Epidemiology, vol. 27, pp. 90-99, 2017/01/01, 2017.

[31] K. Sangkharat, P. Fisher, G. N. Thomas, J. Thornes, and F. D. Pope, "The impact of air pollutants on ambulance dispatches: A systematic review and meta-analysis of acute effects," Environmental Pollution, vol. 254, p. 112769, 2019/11/01/ 2019.

[32] Resolución No. 2254 del 01 de noviembre de 2017, "Por la cual se adopta la norma de calidad del aire de ambiente y se dictan otras disposiciones", Ministerio de Ambiente y Desarrollo Sostenible, Colombia.

[33] G. Pulido, y W. Costa, "Geopolítica y petróleo en el Mar Caribe: la tensión entre Colombia y Nicaragua," Revista Geográfica de América Central, vol. 1, No. 60, pp. 87-116, 2018.

[34] M. C. Díaz-granados, G. E. Francés, y J. Valdés, "Petróleo y geopolítica: Gobernanza Por Otros Medios," Transformaciones en los mercados energéticos, 2015, 83-98.

[35] A. Romero, y M. Vera, "Economía política del petróleo," Criterio Libre, vol. 16, no. 28, pp. 123-146, 2018.

[36] Y. Vargas, y N. León, "Exploración y explotación de petróleo: enfoque geográfico de los efectos ambientales en el Piedemonte llaner," Perspectiva Geográfica, vol. 21, no. 2, p. 199, 2017

[37] L. Sarmiento, "Petróleo, paz inconclusa y nueva lógica del conflicto" Ciencia Política, vol. 13, no. 25, pp. 67-92, 2018.

[38] F. Portero Rodríguez, "El petróleo como arma política," ICE, vol. 1, no. 886, oct. 2015.

[39] L. A. Godoy, S. A. Elaskar, F. M., Francisca, M. A. Montoro, R. C. Jaca, S. N. Espinosa, y M. P. Ameijeiras, "Efectos de desastres naturales y accidentes sobre infraestructura y medio físico en sistemas de almacenamiento y transporte de petróleo," Revista De La Facultad De Ciencias Exactas, Físicas Y Naturales, vol. 6, no. 2, pp. 21-34, 2019.

[40] A. Olaya, y M. Rojas, "Tendencias investigativas del conflicto socioambiental por exploración petrolera," Entre Ciencia e Ingeniería, vol. 13, no. 25, pp. 52-58, 2019.

[41] E. Gudynas, "Los nudos del petróleo en Colombia: ambiente y ciencia, política y democracia," Palabras Al Margen, no. 122, pp. 1-11, 2018.

[42] E. Marin, "Fracking" en Colombia: un Estudio sobre su Constitucionalidad y Legalidad," Facultad de Derecho, Universidad Católica de Colombia, Bogotá, Colombia, 2015.

[43] V. Bacchetta, "Geopolítica del fracking Impactos y riesgos ambientales," Nueva Sociedad, no. 244, pp. 61-73, 2013.

[44] D. Naranjo, "Técnicas, normativa y recomendaciones para la gestión ambiental de la aplicación de la Fractura Hidráulica (fracking) en Colombia," Facultad de Estudios Ambientales y Rurales, Pontifica Universidad Javeriana, Bogotá, Colombia, 2016.

[45] N. Sánchez, "El derecho fundamental a un medio ambiente sano en el marco de la explotación de recursos naturales no renovables: un estudio del fracking en Colombia," Facultad de Derecho, Universidad Católica de Colombia, Bogotá, Colombia, 2016.

[46] I. Salcedo, "Impactos ambientales del fracking analizado desde la experiencia internacional de Estados Unidos," Facultad de Derecho, Universidad Católica de Colombia, Bogotá, Colombia, 2016.

[47] C. Castro, "Análisis jurídico del marco legal de los hidrocarburos no convecionales y de las implementaciones en el derecho ambiental de la técnica fracking," Facultad de Ciencias Jurídicas, Pontifica Universidad Javeriana, Bogotá, Colombia, 2017.

[48] J. Pinto, y A. J. Idrovo, "Fracking, yacimientos en roca generadora y salud humana: entre la incertidumbre y la precaución," Salud, vol. 51, no. 2, pp. 100-103, 2019.

[49] M. Lemos, y M. M. Pedraza, "La autorización del Fracking en Colombia, ¿una decisión apresurada?," Revista de Derecho Público, no. 35, p. 41, 2015. 
[50] J. Gallego, H. Jaramillo, R. Patiño, "Servicios intensivos en conocimiento en la industria del petróleo en Colombia," 2015.

[51] D. Rosselli, "Reflexiones sobre el fracking," Medicina, vol. 41, no. 2, pp. 93-97, 2019.

[52] R. Fernández, "Hacia una transición en las prácticas comunicativas ante la irreversibilidad del cambio climático, el fin del petróleo fácil y la escasez de recursos," Razón Y Palabra, vol. 19, no. 3, pp. 1-4, 2015.
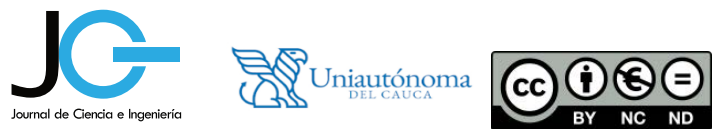